\title{
A comparison of chromatic and achromatic versions of the Stroop color-word test
}

\author{
FREDERICK N. DYER \\ U.S. Army Medical Research Laboratory, Fort Knox, Ky. 40121
}

Achromatic and chromatic versions of the color-word test were given to $25 \mathrm{Ss}$. When the patches were rectangles, achromatic shades were named slightly faster than colors. With interference to naming from the use of incongruent names as stimuli, achromatic shades were named much faster than colors. Interference from incongruent names thus appears to amplify processing time differences and may prove useful for temporal analysis of the visual processing of hue, luminance, and saturation. Extensions of the Stroop test to other perceptual attributes should allow similar analyses of these attributes.

In the typical version of the Stroop color-word tests, a series of color names are printed on a card in colored inks, and the time for naming of the ink colors is compared to the time for naming colors using comparable series of similarly presented rectangular color patches. Typically, the time for naming colors with color names as color patches is at least $50 \%$ greater than that required for simple rectangular patches. A measure of the time for reading color names printed in black ink is also obtained, and, invariably, this is less than the time for naming colors. Jensen \& Rohwer (1966) have provided an extensive review of the Stroop test that includes testing procedures, scoring, research findings, and theoretical considerations.

Treisman \& Fearnley (1969) have presented an insightful analysis of word reading and color naming, the latter both with and without interfering words as color patches. Ss are required to produce words in all three tasks, but in reading, these word responses are matched to other words, whereas in color naming they are matched to colors. In their formulation it is the matching process that is critical, and the matching rate is inversely related to the number of analyzers required for the match (Treisman, 1969). Matching across attributes opens the further possibility of interference and delay from a value on one attribute that is present, although it is to be ignored in making the match.

In the Treisman \& Fearnley (1969) formulation the direction of the match is not critical. If the $S$ were like a chameleon and could also produce colors, the formulation would predict that the color rectangles would now be matched faster than the color names. Such a $S$ would now be prone to interference when he was matching his color responses to words and an "irrelevant" color was present in the color of the ink in which the word was written.
Treisman \& Fearnley (1969) tested their formulation indirectly in a card-sorting task where the cards contained a pair of color or word stimuli, with one of the two usually being both a color and a word stimulus, i.e., a color name written in colored ink. If both stimuli on a card were colors and matching was within the attribute of color, card sorting was very fast. If both stimuli were words and matching was within the attribute of words, card sorting was also very fast. However, when a word was matched to a color or a color was matched to a word, card sorting slowed greatly. When there was a competing "irrelevant" word or color, a further delay in card sorting resulted for cross-attribute matching.

The traditional explanation of results in the Stroop test has been that a strong word-reading response dominates a weaker color-naming response (e.g., Stroop, 1935). Treisman \& Fearnley (1969) have shown that this is less a matter of strength and dominance than a need for an additional perceptual analyzer in color naming beyond the single analyzer that apparently is all that is required for word reading. This illustration, that the initial central correlates of word and color stimuli are different (probably in different cortical locations), would appear to be of extreme importance for our understanding of perception.

Both cross-attribute matching tasks of Treisman \& Fearnley (1969) involved the attribute of color matched with the attribute of words. However, their theoretical formulation suggests that the attribute of color is not critical in the increased time for cross-attribute matching or for the further increase in matching time with interference from a competing attribute value. The naming of other attributes besides color should also be open to interference from a competing word which is from the set of names being used to name the attribute values. Different achromatic shades, in particular, should constitute values on an attribute that would be expected to behave similarly to the attribute of color. Other attributes such as form, spatial position, texture, etc., would also appear to share the potential of color for providing attribute-naming interference situations.

The present study was designed to test whether or not the attribute of achromatic shade would lead to similar increments in time for attribute naming over reading of the attribute names, as occurs for colors, and also to see if further increases in attribute naming time occur when the acromatic shades are combined with words designating incongruous names of these shades. Any differences for achromatic shades from chromatic shades should provide information about perceptual differences for the achromatic and chromatic shades and also about the mechanism of attribute naming interference.

One version of the color-word test developed by Sichel \& Chandler (1969) utilizes only the colors red, green, and blue and the same three color names in incongruous combinations for the interference condition. It uses a plate with the same number of red, blue, and green rectangles for the control color-naming condition. In the present study, similar cards of stimuli were constructed with white, black, and gray substituted for red, blue, and green, with the substitution applying to both words and colors. Naming times for these achromatic cards and also for an ordinary chromatic set of cards were obtained and the results compared to establish whether or not increased naming time for the interference condition occurred for achromatic as well as chromatic versions. One previous study (Smith \& Borg, 1964) found response times for an achromatic version of the Stroop test to be much less reliable than for a chromatic version. The conclusion of the authors was that the achromatic version could not be considered a parallel version of the color-word test, at least for their serial scoring of it. One purpose of this study was to reexamine this question of parallel forms of the Stroop test.

\section{SUBJECTS}

The Ss were 25 males, 17 to 34 years old, who had recently completed basic training in the U.S. Army. All had visual acuity and color vision within normal limits, as measured by the Orthorater.

\section{MATERIALS}

Cards for the chromatic and achromatic interference conditions were prepared, using outline letters (Prestype 2002-24) on white cardboard with the outlines filled in 


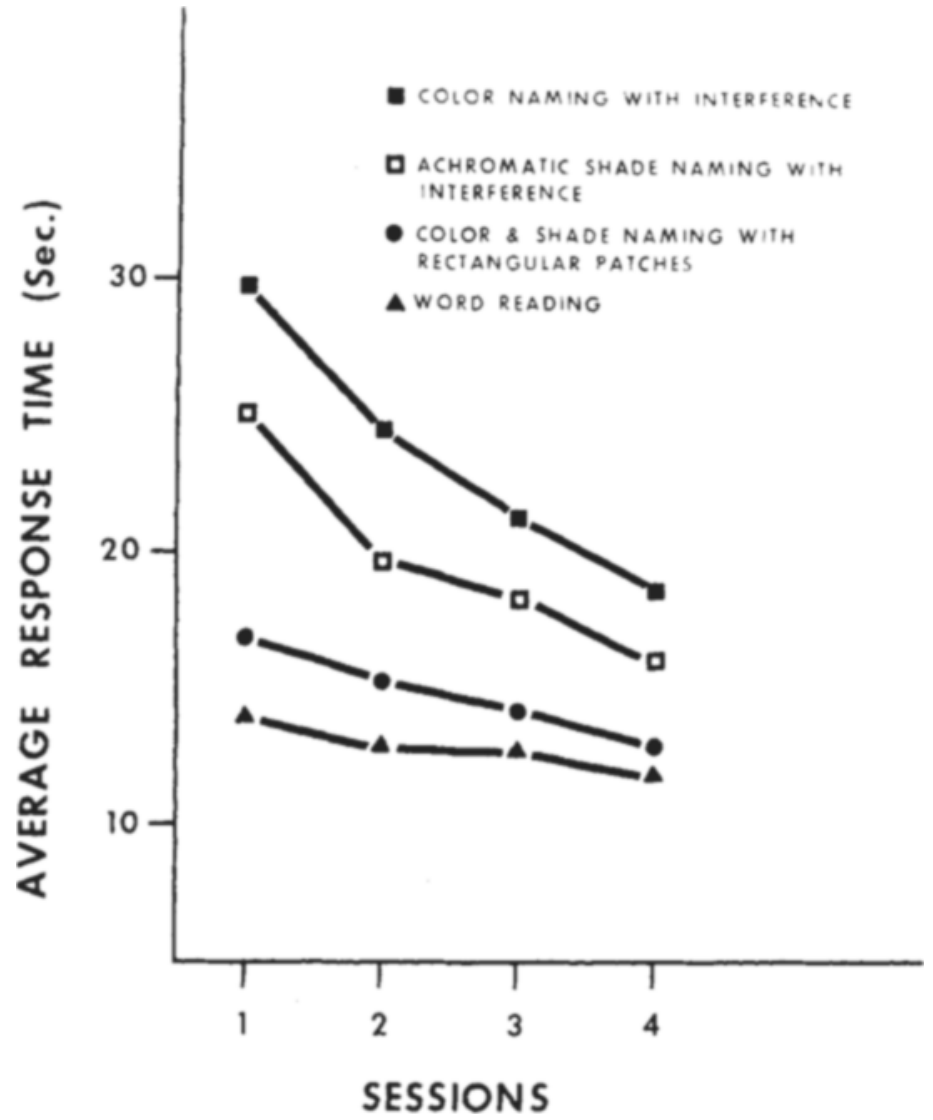

Fig. 1. Average response times for word reading, color naming, and achromatic shade naming for Sessions 1-4.

with the proper colors and shades. The 40 word and color combinations on each of two cards were identical to Plates I and II described in the Sichel \& Chandler (1969) paper. The two corresponding achromatic interference cards were identical in form to these same plates with an isomorphism between gray and green, black and blue, and white and red. This isomorphic relation applied both to words and colors. In addition, a color card with 40 rectangles was constructed with red, blue, and green rectangles arranged with the same order of colors as the ink colors of Sichel and Chandler's Plate V. A similar plate of achromatic rectangles was constructed with gray, black, and white rectangles corresponding to the green, blue, and red rectangles of the color rectangle card. Finally, to investigate reading time for the color names and names of the achromatic shades, two sheets of color names and two sheets of achromatic shade names were typed in black with the words corresponding to the words of Plates I and II of Sichel and Chandler. For the achromatic names the same isomorphism (red for white, blue for black, and green for gray) used on the interference cards was used.

$$
\text { PROCEDURE }
$$

The Ss were tested individually with response times for the entire plate of 40 stimuli recorded with a stopwatch to the nearest 10 th of a second. Each $S$ was tested in four sessions, one session per day on 4 successive days. Eight stimulus plates were presented at each session: one color-name reading plate, one achromatic shade name reading plate, one plate of colored rectangles, one plate of achromatic shaded rectangles, two plates of color-name color patches, and two plates of achromatic shade patches that were names of achromatic shades. At each session for each $S$, a different random order of the eight plates was used. Time between plates was minimal, representing only the time necessary to record data and present the new stimulus plate. Instructions stressed both speed and accuracy, with $S$ required to correct any errors.

\section{RESULTS}

The data were analyzed in a two-way repeated measures analysis of variance, with one factor corresponding to the four sessions and an eight-level factor corresponding tis the eight plates used in each session. Highly significant differences appeared for the main effect of stimulus plates, $F(7,168)=67.3, p<.001$. The mean reading time for color names was $12.92 \mathrm{sec}$; for achromatic shade names it was $12.63 \mathrm{sec}$. Average naming time for the colored rectangles was $15.02 \mathrm{sec}$ and for achromatic shaded rectangles, 14.52 sec. With interference, the average color naming time for Plate I was $23.54 \mathrm{sec}$ and for Plate II, $23.55 \mathrm{sec}$. Achromatic shade naming with interference required an average time of $19.83 \mathrm{sec}$ for Plate I and $19.73 \mathrm{sec}$ for Plate II. A Newman-Keuls test of ordered means indicated that all two-way combinations of average response times from different pairs, as listed above, differed from each other at the .01 level of significance. Members of each pair, however, did not differ between themselves at the .05 level.

The most noteworthy of these significant differences are (1) the longer color naming times for achromatic interference plates over the time for the achromatic plate with rectangular patches, and (2) the longer time for color interference plates over achromatic interference plates.

Color naming became increasingly faster from Session 1 to Session 4, particularly for the interference plates. The main effect of sessions was highly significant, $F(3,72)=36.1, p<.001$, as was the interaction of Plates by Sessions, $F(21,504)=11.4, p<.001$. Figure 1 illustrates this interaction. The four curves in Fig. 1 correspond to the averages at each sessions of the two-word reading scores, the averages of the attribute-naming scores for achromatically shaded and colored rectangles, the averages for the two interference condition plates with color naming, and the average of the two interference condition plates with naming of achromatic shades

\section{DISCUSSION}

Despite their formal identity in the Treisman \& Fearnley (1969) analysis, significant differences in interference appeared for the chromatic and achromatic versions of the test. This suggests a longer processing time for the attribute of color than for achromatic shades. The Newman-Keuls test indicated that the difference in color naming with rectangles and achromatic shade naming with rectangles approached significance at the .05 level, with the time for colored rectangles being $.5 \mathrm{sec}$ longer. Seventeen of $25 \mathrm{Ss}$ had longer times for naming the colored rectangles than for naming the achromatic ones, and this differs significantly from a 50/50 split $(\mathrm{p}=.054)$. This probable slower processing of color information 
relative to the processing of the achromatic shades could allow more opportunity for interference from the competing word in color naming than in the naming of achromatic shades. A recent study (Dyer, 1970) has indicated that slow color processing relative to word processing increases interference to color naming in the Stroop test. The similar association of reduced interference with faster processing of achromatic shades in the present study lends further support to an inverse relationship existing between attribute-naming interference and the processing rate of the attribute.

Whereas the differences in naming time for chromatic and achromatic rectangles are small and only barely significant, the difference between achromatic and color versions of the test in the interference condition $(3.8 \mathrm{sec})$ is over 10 times the standard error of this difference. Thus, a barely detectable difference in processing rates for achromatic and chromatic shades by one measure, namely, speed of color naming with rectangular patches, is vastly amplified in the relative attribute-naming speeds with the addition of interference to naming by a competing word. This property of amplifying differences in processing time would appear to make the Stroop test a useful tool for study of the effects of such variables as hue, saturation, and luminance on the rate of peripheral and central processing of color stimuli. Extensions of the Stroop test, such as the present achromatic version, would seem to hold similar potential for study of variables related to the particular attribute being named.

In conclusion, some discussion of the comparability of interference on the chromatic and achromatic versions of the Stroop test is in order. Interference scores were calculated for each $S$ by determining the difference between average naming times for the interference plates and average times for naming with the rectangular patches. Two such average interference scores for all sessions were obtained for each $S$, one for the achromatic and one for the chromatic versions of the test. Interference scores on one version correlated a remarkable .94 with interference scores on the other version. When three $\mathrm{Ss}$ showing extreme interference on both versions of the test were eliminated, the correlation dropped to .71 , which is still a very strong relationship. This indicates that, for purposes of assessing interference, either test is suitable, since such a high correlation implies high reliability for each. An important consequence of this is that a population which includes color

delectives who cannot easily differentiate the colors of the ordinary Stroop test can be readily compared on their performance by using an achromatic version for the group rather than the usual test.

\title{
REFERENCES
}

DYER, $F$. N. Word reading, color naming and Stroop interference as a function of background brightness. USAMRL Report No. 889, August 17, 1970.

JENSEN, A. R., \& ROHWER, W. D., JR. The Stroop color-word test: A Review. Acta Psychologica, 1966, 25, 36-93.

\section{Errors and latency of response as a function of order of presentation of tactile-visual stimuli in concept identification*}

\author{
AARON WOLFGANG† \\ The Ontario Institute for Studies in Education, Toronto \\ and \\ University of Toronto, Toronto, Canada
}

A task using plastic geometric objects was designed to study concept identification through the tactile sense. The overall results of this study showed that errors and time to solution were similar in classifying visual and tactile information, but response latency was significantly longer in tactile than in visual concept identification. Also, there is some suggestive evidence that visual concept identification was facilitated with practice on a tactual task whereas tactual concept identification was not facilitated by practice on a visual task.

It has been pointed out by the anthropologist Hall (1966) that America is a touchless society. Children are often told by their parents, "You can look, but don't touch," and schools support this idea. The schools, except Montessori, offer very little training in utilizing the tactile sense. As far back as the 1700 s, Berkeley contended that an infant learns a ball is round not by looking at it but by feeling it. Until the child actually handled the ball, the sight of its roundness was meaningless. Touch educated vision.

Most studies and theories in concept identification are based on responses to visual stimuli. In contrast, studies exploring parameters that affect tactile concept identification are almost

* This research was partially supported by the Defense Atomic Support Agency, RMD 1.153 and was done while the author was at the Walter Reed General Hospital and Walter Reed Institute of Research. The author is grateful for the assitance of Miss Juanita Weaver and Daniel Hoeschen. †Requests for reprints should be sent to Aaron Wolfgang, Department of Applied Psychology. The Ontario Institute for Studies in Education, 252 Bloor Street West, Toronto 181 , Ontario, Canada. totally lacking. There have been only a few Es who have attempted to compare learning rates in concept identification using such different sensory modalities as audio-visual (Bulgarella \& Archer, 1962; Haygood, 1965; Lordahl, 1961). The present study extends a prior one by Wolfgang (1969), where visual and tactile concept learning were compared. The overall aim of the present study was to explore transfer effects from visual to tactile and from tactile to visual concept learning. In Berkeley's terms, this experiment attempts to answer the question, "Does touch educate vision more than vision touch?"

\section{METHOD}

All 22 Ss were military duty personnel assigned to Walter Reed Army Medical Center. Their overall mean age was 23.91 years (range: 19-33), and mean education was 12.59 years (range: 11-16).

Ss participated in a 2 by 2 repeated measures design that included two orders of stimulus presentation (visual first, tactile second, or tactile first and visual second) and two modes of stimulus presentation (visual-tactile); there was always one relevant 